\title{
The CMS ECAL Upgrade for Precision Crystal Calorimetry at the HL-LHC
}

\author{
Nancy Marinelli*ł \\ University of Notre Dame, IN, USA \\ E-mail: nancy.marinelliecern.ch
}

The Compact Muon Solenoid Experiment (CMS) is operating at the Large Hadron Collider (LHC) with proton-proton collisions at $13 \mathrm{TeV}$ center-of-mass energy and at a bunch spacing of $25 \mathrm{~ns}$. New further challenging running conditions for CMS are expected after the High-Luminosity upgrade of the LHC (HL-LHC). The CMS electromagnetic calorimeter (ECAL) will be upgraded to sustain the hardened environment. The design and R\&D studies for the ECAL upgrade are presented together with first test-beam studies. Particular challenges at HL-LHC are the harsh radiation environment, the increasing data rates and the extreme level of pile-up events, with up to 200 simultaneous proton-proton collisions. Precision timing can be exploited to reduce the effect of the pile-up. Time resolution measurements carried out during test beams are shown. Plans are also shown for R\&D for the new readout and trigger electronics, which must be upgraded due to the increased trigger and latency requirements at the HL-LHC

EPS-HEP 2017, European Physical Society conference on High Energy Physics, 5-12 July 2017, Venice, Italy

* Speaker.

${ }^{\dagger}$ On behalf of the CMS Collaboration 


\section{The CMS legacy electromagnetic calorimeter}

The CMS lead-tungstate crystal ECAL was designed to be compact, hermetic, fine grained $(\Delta \eta \times \Delta \phi \simeq 0.0174 \times 0.0174)$, low noise $(50 \mathrm{MeV}$ in the Barrel) and to provide an energy resolution, $\sigma_{\mathrm{E}} / E$ of about $1 \%$. All of this with appropriate radiation tolerance [1]. This calorimeter played a pivotal role in the discovery of the Higgs boson in the two-photon decay channel as well as in the initial studies of the boson properties [2], [3]. In the future phase, the HL-LHC will provide unprecedented luminosity, both instantaneous $\left(5 \times 10^{34} \mathrm{~cm}^{-2} \mathrm{~s}^{-1}\right)$ and integrated $\left(250 \mathrm{fb}^{-1}\right.$ per year for about ten years), opening additional discovery windows, particularly the di-Higgs boson production, and allowing for precision studies of the Higgs boson. Electrons and photons will continue to be crucial for the future CMS physics program. The HL-LHC will provide us with a much larger amount of data; the price to pay, however, for a very high luminosity will be the much higher average number of overlapping (pileup) interactions as well as a level of radiation about six times higher than at present. The CMS electromagnetic calorimeter will have to undergo an upgrade, which will allow it to keep pace with the new very different conditions and ensure the same good performance obtained so far.

\section{The electromagnetic calorimeter at the HL-LHC}

The much higher radiation level expected during the HL-LHC operation phase will induce severe transparency loss to the $\mathrm{PbWO}_{4}$ crystals in the endcaps, which will therefore be completely replaced by a new subdetector [4]. The crystals in the barrel (EB), however, are expected to operate well until the end of the HL-LHC program with at most 50\% transparency loss. There is thus no need for replacement of the sensitive detector material, but steps are in any case required in order to cope with the new LHC conditions.

The major motivation for the upgrade is the expected large increase of the CMS Level 1 trigger rate, which will step up from the present $150 \mathrm{kHz}$ to $750 \mathrm{kHz}$ associated to an increased Level 1 CMS latency which is expected to double, from $6.4 \mu s$ to $12.5 \mu \mathrm{s}$. Both front-end and back-end electronics, which presently serve the EB will not be able to work under such conditions. Further effects, which need to be mitigated, are expected: the increase of the dark current in the Avalanche Photo Diodes (APD) [5] due to damage from radiation will lead to larger noise; the larger number, which scales with the instantaneous luminosity, of anomalous signals due to direct interaction of hadrons in the APD bulk; and finally the increased level of pileup interactions. The overall goal is to refurbish the EB electronics to keep its performance stays at the same level of Run I and Run II.

In the upgrade phase the geometrical arrangement of crystals into five-by-five matrices will be left untouched, as well as the APDs and the motherboards (Fig. 1); the very front-end and front-end electronics will be replaced, as well as the off-detector electronics and the LVR cards.

The new very-front-end electronics include a dual-gain Trans-Impedence Amplifier (TIA) with a $50 \mathrm{MeV}$ to $2 \mathrm{TeV}$ dynamic range followed by a dual $\mathrm{ADC}$, which will digitize to 12 bits at a frequency of $160 \mathrm{MHz}$ (Fig.2). Each front-end card will provide the full readout of five very-front-end cards. The Level 1 pipeline will be moved off-detector, where the $12.5 \mu$ s Level 1 trigger latency can be easily accommodated. The generation of trigger primitives, which presently produced in the front-end, will be moved to the back-end, leaving freedom for maximum granularity and future 
development of algorithms. The connections between the front and the back end will be assured by new high-speed links (lpGBT and Versatile links) which can sustain a bandwidth of up to $10 \mathrm{~Gb} / \mathrm{s}$.

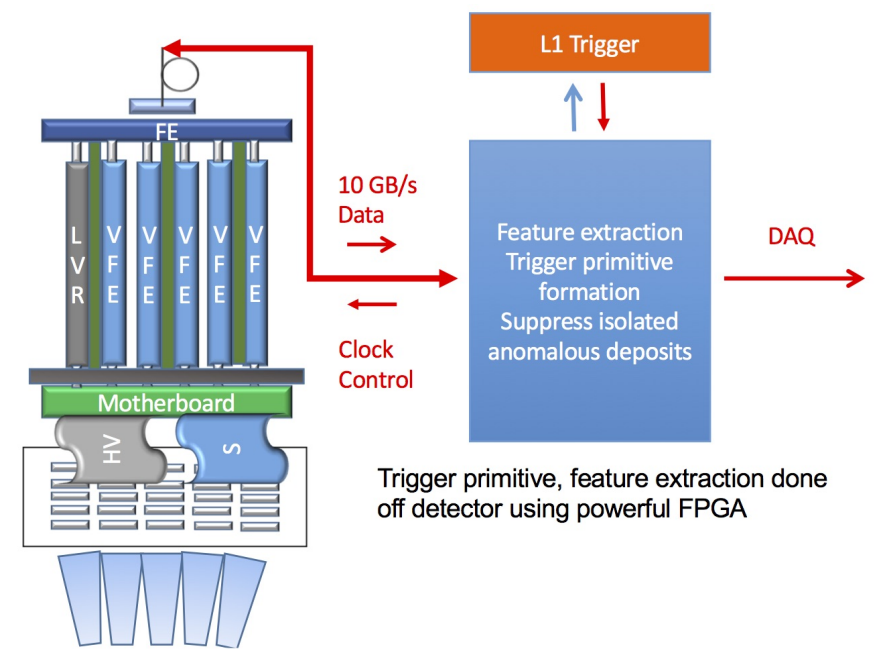

Figure 1: Block diagram for the upgrade of the ECAL barrel electronics.

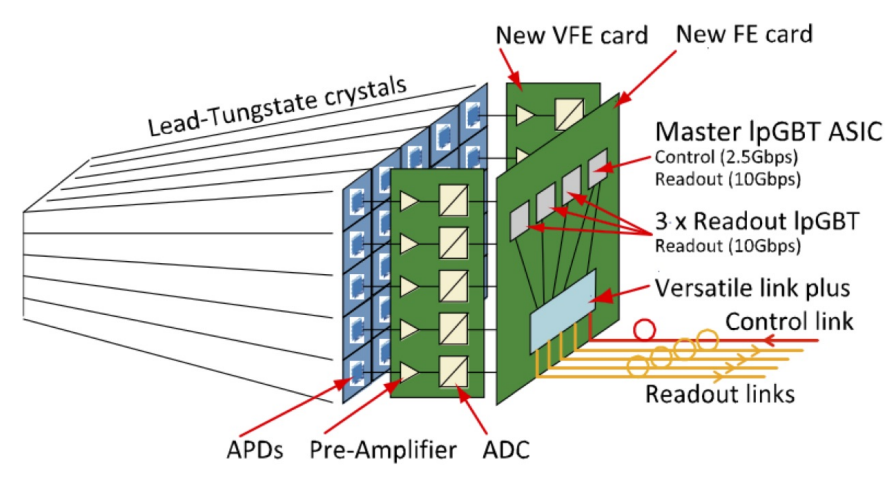

Figure 2: Layout of the upgrade EB electronics architecture.

The integrated luminosity-dependent increase of the APD dark current is expected to result in a factor ten increase in noise after $3000 \mathrm{fb}^{-1}$. Because the dark current strongly depends on the operating temperature, the plan is to reduce the operating temperature from the present $18^{\circ} \mathrm{C}$ to $8^{\circ} \mathrm{C}$ and thus reduce the noise by $35 \%$ as shown in Fig. 3 .

The TIA will have a short shaping time, which will help reduce the contribution to the signal from the out-of-time pileup events. Also it will make possible the distinction between genuine scintillation signals in the APD and anomalous "spike" signals. The latter are produced by hadrons occasionally hitting the bulk of the APDs and can exceed $100 \mathrm{GeV}$. Since their rate is proportional to the instantaneous luminosity, they need to be suppressed to avoid a Level 1 rate that would otherwise be unsustainable. The signal from a spike is intrinsically narrower than a genuine signal (Fig. 4). The single channel readout and the high-frequency sampling (160MHz) and the event- 


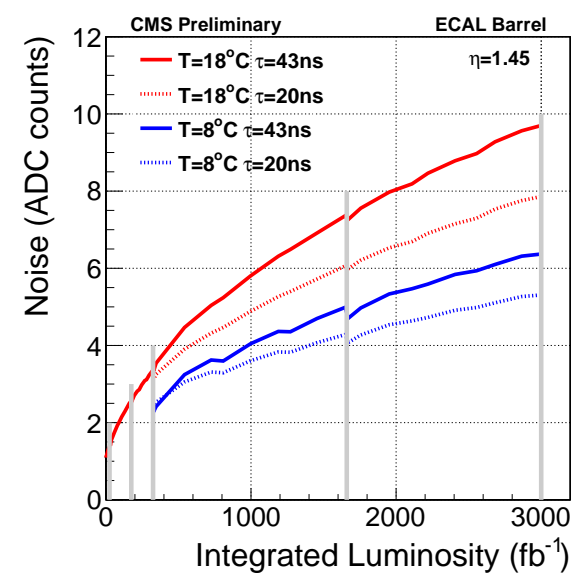

Figure 3: Expected noise in EB as a function of the integrated luminosity and for two different temperatures (present and future planned) as well as with two different peaking times.

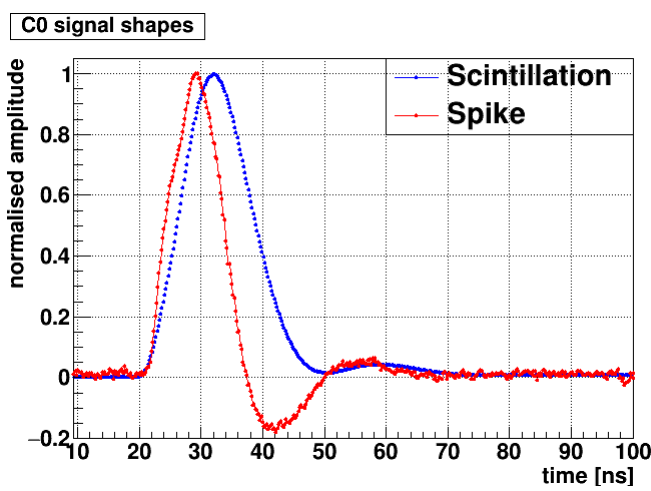

Figure 4: The signal from an anomalous spike (light grey) is narrower the genuine signal form the scintillation (dark grey).

by-event and channel-by-channel signal analysis would allow for the disentangling. This would represent a significant improvement with respect to what is presently done, where a topological algorithm, based on the $5 \times 5$ crystal matrix, is applied. The latter would be too vulnerable to pileup effects and, as simulations show, the spike rejection efficiency would become unacceptably small.

Identification of the primary signal vertex within $1 \mathrm{~cm}$ is vital for the Higg boson mass measurement [2] in the decay to two photons. One of the major effects deriving from the increased pileup will be the largely reduced identification efficiency of the primary vertex. This problem can be partially overcome with high-precision timing in the EB which will be possible thanks to the new VFE. The short shaping time jointly with the signal sampling at $160 \mathrm{MHz}$ should make a time resolution of about 30ps possible for electromagnetic energy deposition of several tens of $\mathrm{GeV}$ as shown by the first test-beam results (Fig. 5). This precision timing would result in a primary vertex efficiency comparable to that of LHC Run I and II (about 80\%). Also it would help removing the in-time pileup contributions to the neutral energy in the clusters in the EB. 


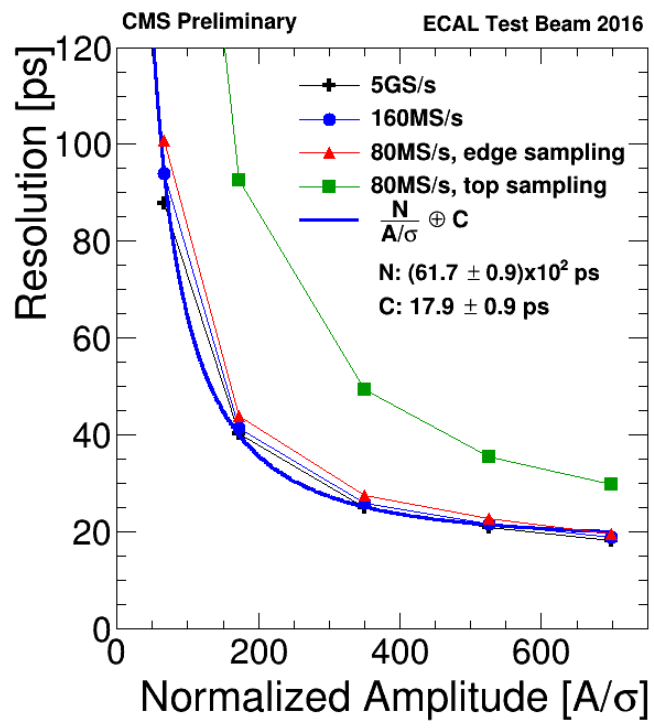

Figure 5: Timing resolution function of the noise-normalized amplitude obtained in a test beam using prototype TIA electronics. A resolution of about $30 \mathrm{ps}$ is reached for an amplitude/noise $\sim 240$ which corresponds to an energy deposition of $25 \mathrm{GeV}$ at the beginning of HL-LHC (noise $\approx 100 \mathrm{MeV}$ ) and $50 \mathrm{GeV}$ at the end of HL-LHC (noise $\approx 200 \mathrm{MeV}$ ).

\section{Summary}

The future challenging HL-LHC phase will require for the ECAL barrel: a longer data pipeline and larger bandwidth; a more powerful filter at trigger level against anomalous "spike" signals; the mitigation of increased APD noise as well as precision timing for primary vertex determination and pileup energy subtraction. The accomplishment of all these goals will require the design and replacement of the front and very-front end electronics as well as new powerful off-detector boards for processing high bandwidth/granularity data. The ECAL barrel will hence undergo a full refurbishment and recommissioning during the LHC third long shutdown in the years 2024-2025.

\section{References}

[1] CMS Collaboration, The CMS electromagnetic calorimeter project: Technical Design Report, Technical Design Report CMS. CERN, Geneva, 1997.

[2] V. Khachatryan et al., Observation of the diphoton decay of the higgs boson and measurement of its properties, The European Physical Journal C 74 (2014), no. 10, 1-49.

[3] CMS Collaboration, Measurements of properties of the Higgs boson in the diphoton decay channel with the full 2016 data set, CMS Physics Analysis Summary CMS-PAS-HIG-16-040, 2017, http://cds.cern.ch/record/2264515.

[4] A. Steen on behalf of the CMS Collaboration, The CMS HGCAL detector for HL-LHC upgrade, these proceedings.

[5] D. Bailleux et al., Hamamatsu APD for CMS ECAL: Quality insurance, Nucl. Instrum.Meth. A518 (2004) 622-625, doi:10.1016/j.nima.2003.11.102 